\title{
Therapy for penicillin-resistant Streptococcus pneumoniae
}

Penicillin-resistant pneumococci have been recognised since the late 1970s and continue to increase in incidence worldwide. The incidence in children in Soweto, South Africa, has stabilised at c. $40 \%$. Production of $\beta$-lactamase has not been demonstrated and resistance to $\beta$-lactam antibiotics results from changes in the penicillin-binding proteins in the bacterial cell wall. The chromosomal mutations coding for resistance are stable, so it is possible for resistant isolates to spread and cause disease in individuals without prior exposure to antibiotics.

Resistance is subdivided according to the MIC of penicillin. Pneumococci for which the MIC of benzylpenicillin is $<0.1 \mathrm{mg} / \mathrm{L}$ are defined as susceptible, those for which the MIC is $0 \cdot 1-1.0 \mathrm{mg} / \mathrm{L}$ as intermediately resistant and those for which the MIC is $>1.0 \mathrm{mg} / \mathrm{L}$ as highly resistant. Disease presentation is not altered when the infecting organisms are penicillin resistant except that resistant organisms are more likely to be isolated from patients with underlying immune compromising conditions. ${ }^{1}$

\section{Meningitis}

Penicillin therapy is frequently ineffective in meningitis caused by pneumococci exhibiting intermediate resistance, because of the relatively low concentrations of penicillin achievable in the cerebrospinal fluid (CSF). Therapy with an extended spectrum cephalosporin is usually successful, but cephalosporin-resistant pneumococci have been reported in the USA since $1991 .^{2}$ The definition of cephalosporin resistance has been modified over the past few years: an MIC $\leqslant 0.25 \mathrm{mg} / \mathrm{L}$ is now regarded as indicating full susceptibility; $0.5-1.0 \mathrm{mg} / \mathrm{L}$ as moderately susceptible; and $\geqslant 2.0 \mathrm{mg} / \mathrm{L}$ as resistant. ${ }^{3}$ The MICs of all $\beta$ lactam antibiotics generally increase in parallel with those of penicillin. MICs of cephalosporins for intermediately and highly resistant pneumococci are higher than those of penicillin-susceptible strains. Therefore, it is essential that cephalosporin MICs are determined for all penicillin-resistant isolates from CSF. Several techniques are available for determining MICs; the Etest is attractive because it is rapid, simple and relatively inexpensive compared with other methods. ${ }^{4}$

The best regimen for the treatment of cephalosporin-resistant pneumococcal meningitis is yet to be determined. The current recommendation, in areas where cephalosporin resistance is a problem, is to combine an extended spectrum cephalosporin with vancomycin or rifampicin as empiric therapy. ${ }^{5-6}$ Once MICs of cephalosporins are available, the therapy should be revised. The cephalosporin resistance rate in South Africa is low $(<2 \%)$; therefore we continue to use a cephalosporin alone as empiric therapy for suspected pneumococcal meningitis. However, if a penicillin-resistant pneumococcus is isolated from the CSF and the cephalosporin MIC is not available within $36 \mathrm{~h}$ of the start of therapy, the lumbar puncture is repeated to confirm that the CSF has been sterilised.

\section{Pneumonia and sepsis}

There is no convincing evidence that therapy with penicillin or an equally active $\beta$-lactam antibiotic is ineffective in pneumonia or sepsis due to pneumococci that are intermediately susceptible to penicillin. Reports of poor outcome in adult patients with sepsis or pneumonia usually included patients who were critically ill or had an underlying disease. The clinical condition of the patient at the start of therapy is probably a more important determinant of outcome than the sensitivity of the organism to penicillin. Recent unpublished data from our hospital suggest that infections outside the central nervous system respond to standard dose ampicillin or penicillin therapy as well as those with fully susceptible pneumococci. The MIC breakpoint above which penicillin therapy is likely to be ineffective for non-meningeal infections is unknown, but is probably $\geqslant 4.0 \mathrm{mg} / \mathrm{L}$, and such strains are rare.

If cephalosporins are to be used for suspected pneumococcal pneumonia or sepsis, compounds that are more active than penicillin are likely to be effective irrespective of the degree of penicillin resistance. By contrast, cephalosporins that are poorly active against penicillin-resistant pneumococci may be ineffective even for strains that are intermediately resistant (table). ${ }^{7}$

At present, we recommend no special therapy for pneumococcal pneumonia but do caution against the use of poorly active cephalosporins. If a penicillinresistant pneumococcus is isolated the clinician should

Table. In-vitro activity of selected $\beta$-lactam antibiotics relative to penicillin

\begin{tabular}{lll}
\hline More active & Equally active & Poorly active \\
\hline Imipenem & Ampicillin & Oxacillin \\
Meropenem & Amoxycillin & Cefixime \\
Cefotaxime & Piperacillin & Cefaclor \\
Ceftriaxone & Cefpodoxime & Ceftazidime \\
Cefpirome & Cefuroxime & Cefoxitin \\
& & \\
\hline
\end{tabular}


be guided more by the clinical response of the patient than by the in-vitro susceptibility of the organism. The laboratory should be aware of the limited clinical significance of intermediate penicillin resistance.

\section{Otitis media}

Penicillin-resistant pneumococci are a frequent cause of treatment failure in otitis media in some areas. ${ }^{8}$ To date most strains associated with treatment failure were highly resistant to penicillin and were often resistant to other oral agents such as erythromycin (and the newer macrolides) and co-trimoxazole. No oral cephalosporin is more active than amoxycillin against pneumococci and amoxycillin remains the drug of choice for the empiric therapy of otitis media. Co-amoxiclav is not more effective than amoxycillin alone, but does have the advantage of being active against $\beta$-lactamase producing organisms. Oral cephalosporins are also active against $\beta$-lactamase-producing organisms but otherwise offer no advantage over amoxycillin as empiric therapy of otitis media. In areas where resistant pneumococci are common, tympano-

\section{References}

1. Friedland IR, Klugman KP. Antibiotic-resistant pneumococcal disease in South African children. Am J Dis Child 1992 146: 920-923.

2. Bradley JS, Connor JD. Ceftriaxone failure in meningitis caused by Streptococcus pneumoniae with reduced susceptibility to beta-lactam antibiotics. Pediatr Infect Dis $J$ 1991; 10: $871-873$.

3. Jorgensen JH, Swenson JM, Tenover FC, Hindler JA, Murray PR. Development of quality control interpretive criteria of antimicrobial susceptibility testing of Streptococcus pneumoniae. In: Program and abstracts of the 33rd Interscience Conference on Antimicrobial Agents and Chemotherapy, New Orleans, October 17-20, 1993. Washington, DC, American Society for Microbiology, 1993: 167.

4. Jacobs MR, Bajaksouzian S, Appelbaum PC, Bolmström A. Evaluation of the E-test for susceptibility testing of pneumococci. Diagn Microbiol Infect Dis 1992; 15: 473478 . centesis should be considered in cases failing oral therapy. Rational therapy can then be based on the susceptibility of the isolated organism. Intramuscular ceftriaxone therapy (as a single dose) is likely to be effective in cases failing oral therapy because of its activity against penicillin-resistant pneumococci and $\beta$-lactamase-producing organisms. ${ }^{9}$

Pneumococci that exhibit intermediate resistance to penicillin are likely to be of clinical significance only in meningitis. The clinical significance of the current definition of cephalosporin resistance is yet to be verified but is likely to be important only in meningitis and otitis media. All institutions should document and report their experience with therapy of penicillinresistant pneumococcal infections so that treatment protocols can be formulated. It is vital that reports of treatment outcome include controls (patients with penicillin-susceptible infections) in order to eliminate potential bias.

\section{S. A. Strachan and I. R. Friedland Department of Paediatrics, Baragwanath Hospital and the University of the Witwatersrand, PO Bertsham 2013,} South Africa

5. Friedland IR, Paris M, Ehrett S, Hickey S, Olsen K, McCracken GH. Evaluation of antimicrobial regimens for treatment of experimental penicillin- and cephalosporin-resistant pneumococcal meningitis. Antimicrob Agents Chemother 1993; 37: $1630-1636$

6. Klugman KP, Bradley JS, Friedland IR. Combination therapy of third-generation cephalosporin-resistant pneumococcal meningitis. In: Abstracts and proceedings of the 5th Joint Congress of the Sexually Transmitted Diseases and Infectious Diseases Societies of Southern Africa, 20-24 May 1995, Durban, South Africa, p. 69.

7. Liñares J, Alonso T, Pérez JL et al. Decreased susceptibility of penicillin-resistant pneumococci to twenty-four $\beta$-lactam antibiotics. J Antimicrob Chemother 1992; 30: 279-288.

8. Klein JO. Microbiologic efficacy of antibacterial drugs for acute otitis media. Pediatr Infect Dis $J$ 1993; 12: 973-975.

9. Green SM, Rothrock SG. Single-dose intramuscular ceftriaxone for acute otitis media in children. Pediatrics 1993; 91: $23-30$ 